Original Article

\title{
PHARMACOECONOMIC EVALUATION FOR DENGUE VACCINE PRICING FOR MALAYSIA: TOWARDS AFFORDABILITY, COST EFFECTIVENESS AND SUSTAINABILITY
}

\author{
HOCK LYE KOH ${ }^{a}$, SU YEAN TEH ${ }^{b^{*}}$, NOORLIZA M. NOORDINc, LOKMAN H. SULAIMAN ${ }^{d}$
}

aSchool of Mathematical Sciences and Jeffrey Sachs Center on Sustainable Development, Sunway University, Jalan Universiti, Bandar Sunway, 47500 Selangor Malaysia, bSchool of Mathematical Sciences, Universiti Sains Malaysia, 11800 Pulau Pinang, Malaysia, cHead, National Public Health, Ministry of Health, 59000 Kuala Lumpur, Malaysia, dDeputy Director-General of Health, Ministry of Health, 59000 Kuala Lumpur, Malaysia

Email: syteh@usm.my

Received: 05 Feb 2017 Revised and Accepted: 18 Apr 2017

\begin{abstract}
Objective: Malaysian health ministry is currently evaluating the licensing of dengue vaccine Dengvaxia using a set of criteria including safety, efficacy and cost-effectiveness. The purpose of this research is to formulate a model framework to assist transparent decision-making.
\end{abstract}

Methods: To quantify the cost-effective price of the vaccine, pharmacoeconomic evaluation models are commonly used. We review in this paper dengue economic burdens and pharmacoeconomic models, with particular reference to cost-effectiveness.

Results: A major weakness in current pharmacoeconomic models is the omission of the cost of vaccine production, which is two orders of magnitude lower than the market price. This paper proposes an inclusive model that yields a highly cost-effective threshold price of dengue vaccine at USD 5 per dose for Malaysia.

Conclusion: At USD 5 per dose, Dengue vaccine will be fair, Affordable and sustainable.

Keywords: Pharmacoeconomics, Sustainable dengue vaccine, DALY

(C) 2017 The Authors. Published by Innovare Academic Sciences Pvt Ltd. This is an open access article under the CC BY license (http://creativecommons.org/licenses/by/4.0/) DOI: http://dx.doi.org/10.22159/ajpcr.2017v10s3.21352

\section{INTRODUCTION}

With 2 billion people vulnerable to dengue infections in tropical and subtropical countries where dengue is endemic, about 50 to 100 million dengue infections occur each year leading to 20000 to 30000 deaths annually. The World Health Organization (WHO) advocates the principle of harmonizing equitable cost-effective prevention measures via vaccine and behavioural adaptation with adequate entomological and epidemiological surveillance, coupled with good case management within existing health care systems. Regarding vaccines, several tetravalent dengue vaccine candidates are currently under various phases of evaluation. A recurring concern among these vaccine candidates is the inability to achieve balanced immune responses to all four serotypes. Of these candidates, Dengvaxia has been licensed in more than ten countries and is currently undergoing review in Malaysia. Taking a proactive approach, the authors aim to develop an equitable cost-effective mechanism for pricing dengue vaccine in Malaysia to achieve social equitability and sustained vaccine adoption, although pricing issues are currently not an integral part of the licensing process. To provide an open and transparent mechanism for vaccine pricing to assist the policy decision process, various pharmacoeconomic models have been proposed. Following WHO guidelines, these pricing models invariably attempt to address the cost-effectiveness of dengue vaccine by relating the threshold price to the Gross Domestic Production (GDP) of the nation, where the vaccine is to be priced, taking into consideration other factors including the cost of dengue disease burdens and other costs such as vector control. The multitudes of factors involved in vaccine pricing render these models complicated to understand and complex to apply, as the database required are often incomplete and frequently inconsistent. Yet, the intensification of dengue virus (DENV) transmission outbreaks, with increasing frequency and magnitude, in the recent past decade has imposed a major strain on the public health and national wealth of Malaysia. The need to develop an in-country pricing model for dengue vaccine in Malaysia, following WHO guidelines, is therefore particularly urgent. A fundamental underlining fact is that dengue is caused by four distinct but related viruses (DENV 1-4), which are transmitted primarily by the Aedes aegypti mosquitoes. The Aedes mosquito ecology promotes the cocirculation of these four related virus serotypes, in densely populated and unhygienic urban conditions, causing a heightened risk of sequential infections with increased severity of the disease. The higher risk of severe disease with a second heterotypic DENV is thought to be caused by a phenomenon known as antibodydependent enhancement (ADE) of infection.

However, ADE has not been adequately addressed, and in some cases conveniently ignored, in currently available pricing models. A recurring concern regarding these vaccine candidates is the inability to achieve balanced immune responses to all four serotypes. This omission of risks to ADE implies that current pricing models have not factored in the potential DENV infection costs due to vaccineinduced ADE, which might otherwise vastly reduce the costeffectiveness assumed. Therefore, developers of a dengue vaccine must ensure a tetravalent vaccine that can provide equal protection against all four serotypes. The current version of Dengvaxia fails in this regard and hence needs further improvement before it is entitled to a claim of adequate protection to vaccine users against all four viruses. Nevertheless, because widespread and effective mosquito vector control has been difficult to sustain, a cost-effective and efficacious dengue vaccine is highly desirable in an environment of a persistent dengue epidemic. Despite the apparent effectiveness of vector control programs in reducing vector indices in Singapore before the 1990s, dengue repeatedly resurged in Singapore since the 1990 s, due to a number of factors, including the reduction of the herd protection available in the 1970s and 1980s. Cyclical epidemics have reoccurred since the 1990s, peaking in 2005 when the 
incidence of confirmed dengue was 335 per 100,000 populations. The recent trial experiment in Malaysia using genetically modified male mosquitoes 0X513A produced by Oxitec to suppress wild mosquito population has proven to be unsustainable economically nor logistically [1]. Therefore, safe, efficacious and cost-effective dengue vaccines coupled with sustainable vector control via behavioural adaptation are critically essential in the fight against DENV. Regarding vaccine adoption, it has been proven through various vaccine applications in lower income countries (LIC) that price is the most important factor influencing vaccine introduction and sustained adoption in LICs, once safety and efficacy of the vaccine is adequately assured. This paper proposes a vaccine price that will ensure ready and sustained adoption of dengue vaccine in Malaysia, given its middle-income country (MIC) status. The paper starts with a brief review on economic burdens of dengue disease worldwide to highlight the wide variability in dengue disease burdens within and across nations, due to the wide uncertainty and inconsistency in input parameters used. Because of the large variance in the mosquito populations and equally large variance in the cyclical prevalence of infection, it is a challenging task to conduct vector and dengue surveillance on a scale large enough to allow for meaningful conclusions on disease burden estimation, with and without vaccines. The review reveals that (a) most vaccines are readily accepted and sustainably adopted if they were priced below USD 1 in LIC and that (b) dengue vaccine can be sustainably produced at around USD 0.50 per dose. DENV imposes a major strain on the public health infrastructure that is disproportionately borne by lower middle-income countries (LMIC). Hence, any efforts to mitigate DENV transmission in LMIC should give due consideration to this issue of eliminating global inequity. We then review some pharmacoeconomic models that have been used, for example, in Singapore and in Malaysia, to provide insights on dengue vaccine pricing methodology. We then propose our own in-country evaluation model in which we adopt the strategy of highly costeffectiveness, above being merely cost-effective. The paper will end with our recommendation that a highly cost-effective threshold price of dengue vaccine for Malaysia is USD 5 per dose at the current GDP per capita of USD 11,000 .

\section{MATERIALS AND METHODS}

\section{Economic burden of dengue disease}

To quantify the economic burden of dengue disease, both monetary value and non-monetary public health metrics are used. The quantitative non-monetary measure is known as the Disability Adjusted Life Year (DALY) has been widely used in many studies. DALY is the sum total over the entire target population, of the years of life lost due to each premature mortality plus the years of each healthy life lost due to disability caused by the infection. DALY is typically expressed as DALY per 100,000 populations per year. For example, it has been estimated that the disease burden for Puerto Rico from 1984 to 1994, is estimated to be 65.8 DALY per 100,000 per year [2]. On the worldwide scale, WHO [3] estimated an average of 26.4 DALY is lost per 100,000 populations per year. The estimation of DALY is complicated by many uncertainties in the estimation of a myriad of input. Further, for every reported case of dengue, there are many cases that are not reported. Hence, an expansion factor (EF), with high uncertainty in the estimates, is often used to compensate for the unreported cases. For economic valuations, the estimated DALY is then converted into monetary values, via the nation's GDP per capita, following WHO guidelines. For any country, it is the prerogative of the country government to choose 1.0 DALY to be converted to 1.0-3.0 times GDP per capita. The valuation is called highly cost-effective if 1.0 DALY is converted to $1.0 \mathrm{GDP}$ per capita. The valuation is called cost-effective if 1.0 DALY is converted to 3.0 GDP per capita. There are other economic direct and indirect costs involved too. Hence, the total economic burden of dengue includes loss due to deaths (DALY equivalent), plus direct and indirect medical costs.

Despite the multitude of uncertainty inherent in analysing dengue economic burden, Suaya [4] nevertheless attempted to estimate the average annual aggregate cost of dengue disease burdens over the period 2001-2005 for eight countries: Brazil, El Salvador, Guatemala, Panama, Venezuela, Cambodia, Malaysia and Thailand, based on dengue cases and deaths officially reported to the WHO. The years of premature life lost to dengue are the remaining life expectancies at the age of death based on the country-specific life tables. On average over the whole of the eight countries during the study period, there were a total of $574,000(132,000)$ dengue cases, including 399 (59) deaths, leading to a total of 10,283 DALY, where the standard error is expressed in parentheses. The total cost is USD 238 (41) million, while the average cost per case is USD 417 (21). Average cost per case in terms of days of GDP per capita varied significantly across countries, from $8 \mathrm{~d}$ to $56 \mathrm{~d}$. The estimates also did not include unreported cases, implying the value of EF of 1.0. In most subsequent studies, expansion factors EF of more than 1.0 is used to account for under-reporting of cases, which had a major impact on overall aggregated cost estimates. Another study involving 12 countries in Southeast Asia, covering the decade of 2001 to 2010, recorded an annual average of 2.9 million dengue episodes and 5,906 deaths [5]. The annual economic burden estimated was USD 950 million or about USD 1.65 per capita. The annual number of DALY was 214,000 which is equivalent to 37.2 DALYs per year per 100,000 inhabitants. For Puerto Rico [2], from 2002 to 2010, the aggregate annual cost of dengue disease averaged USD 38.7 million. Including dengue surveillance and vector control activities, the overall annual cost of dengue would be USD 46.45 million (USD 12.47 per capita). To adjust for under-reporting, an expansion factor of 2.42 was used for hospitalized and fatal cases and 10.0 for ambulatory cases. The average cost per death was USD 428,559 overall, with a GDP per capita of USD 16,300 in 2010 for Puerto Rico. This implies a value of 26.29 DALY per premature death due to dengue. These dengue disease economic burden valuations performed in the recent past will provide a sound theoretical foundation and a useful benchmark for the valuation of a highly costeffective price for dengue vaccine in Malaysia in the subsequent sections. This price would also be affordable, fair and sustainable.

\section{Towards affordable dengue vaccine for Malaysia}

As discussed earlier, dengue vaccine remains an important strategy in the control of DENV transmission. Price is the most important factor influencing vaccine introduction and sustained adoption in LMICs, once safety and efficacy are assured. For example, priced at more than several USD per dose, vaccines for rotavirus, conjugated pneumococcal and human papilloma virus had faced significant resistance in their adoption in LICs [6]. On the other hand, meningitis A vaccine, priced at USD 0.50 per dose, was readily adopted throughout the Africa meningitis belt. In the light of such encouraging experiences regarding the acceptance and availability of quality vaccine costing below USD 1, we anticipate a wide availability and ready acceptance of dengue vaccines at about USD 1 for LICs. In the meeting on December 2001 at Vietnam, the Pharmacoeconomics Working Group concluded that at a price per dose of USD 0.50 to the public sector, dengue vaccine would be cost effective for LMICs. Adjusting for inflation, this fig. could be revised to no more than USD 2.00. The resulting cost per DALY saved would be the same order of magnitude as other international public health programmes [7]. Since then, the list of dengue vaccine developers has increased, including GlaxoSmithKline (GSK), In Viragen, Merck, U. S. National Institutes of Health (NIH), and Sanofi Pasteur. Of these, the US-NIH has licensed its live attenuated dengue vaccine candidate to four developing country producers, including Instituto Butantan in Brazil. The Butantan facility can reduce the production cost to about USD 0.20 per dose in 10 -dose vials and about USD 0.70 per dose in single-dose vials [6]. Given this level of dengue vaccine production costs, it is clear that a price which is affordable to consumers and fair to producers for dengue vaccine would be USD 5.00 per dose. As we will see soon, this fig. of USD 5.00 per dose is also a highly cost-effective price threshold for Malaysia, given that its GPD per capita is USD 11,000.

The Developing Countries Vaccine Manufacturers Network (DCVMN) was established in 2000 with the vision and mission of increasing the availability and affordability of quality vaccines to protect against known and emerging infectious diseases. China, India and Brazil are leading the emerging markets in the pace of scientific and technological advances and innovations in vaccine developments. DCVMN played a significant role in supplying quality vaccines at 
affordable prices to UN agencies for the pentavalent vaccines against five diseases-diphtheria, tetanus, pertussis, Hib and hepatitis B. The future offers new opportunities for building vaccine capacities in developing countries to supply high quality and affordable vaccines. However, many MICs, including Malaysia, continue to confront the dilemma of being caught in between LIC and highincome countries (HIC).

The MIC is a group characterised by a wide range of GDP per capita (USD 1,026 to 12,475) [8]. The MICs are not sustainably introducing new life-saving vaccines at the same rate as donor-funded LICs or wealthier HICs. The prices available to MICs for new vaccines are significantly higher than those offered to LIC and will affect the rate of new vaccine introduction and adoption. Without access to the lower prices paid by LICs and donors, MICs are burdened fiscally by the high vaccine prices paid by HICs. A more rapid and sustained uptake of new vaccines in MICs will increase the predictability of demand, increase the level of funding and reduce transaction cost. The MICs are starting, as a group, to lag behind significantly in the sustainable implementation of new life-saving vaccines such as dengue vaccine. This undesirable scenario has to change quickly, in the face of impending outbreaks of vaccine-preventable diseases such as dengue [8]. With this in mind, we propose a new inclusive approach for pharmacoeconomic methodology for dengue vaccine pricing with the goal of making vaccine affordable to Malaysia leading to its sustained adoption.

\section{RESULTS AND DISCUSSION}

\section{New model for dengue pharmacoeconomics}

As the scopes and costs of new vaccines continue to grow in developed nations, and as the developing countries find it increasing difficult to cope, sound economic fundamental is essential in justifying decisions to introduce and sustain vaccination. The majority of studies illustrated that vaccines could be cost-effective at less than USD 1000 per DALY averted in LMIC [9]. Given the low production cost of vaccines in DCVMN, a new analytical framework should be developed in LMIC to evaluate a cost-effective and affordable vaccine price based upon a comprehensive suite of factors that include RandD and production costs and overall economic burden of disease for transparent decision-making. A WHO study found the vaccine to be cost-effective in developed countries at the price of USD 7.64 per dose, weighted average for public and private sector including administrative costs [10]. The price for LMIC should therefore be much lower. Another recent study in Singapore concluded that a dengue vaccine involving three doses and conferring ten years of immunity would be cost-effective at a price threshold of USD 95 per dose, given Singapore GDP per capita of about USD 55,000. However, the authors admitted several limitations in the estimation of the economic and disease burdens [11]. The low efficacy and theoretical increase in risk due to vaccineinduced ADE have not been incorporated in their current pricing model. The low efficacy would require high rates of vaccine coverage to achieve herd immunity during episodes of high DENV transmission with high basic reproduction number $\mathrm{R}_{0}$. With this backdrop, this paper attempts to propose a new pricing model framework for dengue vaccine, based upon five criteria. First, the pricing mechanism adopted in Singapore had arrived at the costeffective threshold price of USD 95 for an assumed effective duration of 10 years**. Given a GDP per capita of about USD 11,000 in Malaysia versus USD 55,000 in Singapore, the GDP parity adjusted threshold price for Malaysia would be USD $95 \times$ USD 11,000/USD $55,000=$ USD 19.00. Second, as an MIC, Malaysia deserves and desires to mandate that the dengue vaccine to be highly costeffective (1.0 DALY equal to 1.0 GDP) as compared to merely costeffective (1.0 DALY equal to $3.0 \mathrm{GDP}$ ) as in the case with the Singapore paper. Given the further price reduction ratio of 3.0 GDP/1.0 GDP = 3.0, the highly cost-effective price for dengue vaccine would be reduced from USD 19.0 to USD 19.0/3.0 or USD 6.3, everything else being equal $[5,7,12]$. Incorporating other uncertainties such as effective duration of less than 10 years**, this cost would be further reduced to USD 5.00, if not lower. Third, in a personal communication [13], a highly cost-effective price in Malaysia for dengue vaccine has been quoted as USD 42.00 per dose for a three-dose regimen. This estimation is based upon a model simulation of dynamic DENV transmission, in which the mosquito transmission biting rate used was 0.67 per day per female mosquito. In our view, the transmission biting rate of 0.67 per day per mosquito is very high as compared to the normal rate universally used i.e. 0.33 per day per mosquito. This doubling of biting rate has conferred vastly inflated and unjustified benefit of vaccine, erroneously leading to higher price. To elaborate further, we resort to mathematical models for dynamic transmission of DENV between human and female mosquito. Equation (1) describes the transmission of the virus from infective vector $I_{v}$ to susceptible human $S_{h}$ to give rise to the infected human $I_{h}$, with a transmission biting rate of $\beta$. Equation (1) is part of a system of eight ordinary differential equations formulated in the traditional SIR model [14, 15] as illustrated in Equation (2). The entire human target population would be quickly infected if a transmission biting rate of $\beta=1.5$ per day per mosquito were to be used [16]. This implies that the rate of $\beta=0.67$ would have produced exceptional and unrealistically high rate of infection and hence would have conferred an unjustifiably inflated benefit to the vaccine by inflation of infections avoided by the vaccine. Using a normal rate of $\beta=0.33$ would reduce the highly cost-effective price from USD 42.00 as suggested in Shafie [13] down to merely USD 7 using concepts in Koh and Teh [1].

$$
\begin{gathered}
\frac{d I_{h}}{d t}=\beta \frac{S_{h}}{N_{h}} I_{v}-\gamma I_{h}-\mu_{h d} I_{h} \\
\frac{d S}{d t}=-\frac{\beta S I}{N} \quad \frac{d I}{d t}=\frac{\beta S I}{N}-\gamma I \quad \frac{d R}{d t}=\gamma I
\end{gathered}
$$

Fourth, there is a serious concern regarding low efficacy $\alpha$ of tetravalent vaccine, with $\alpha$ having value between 0.3 and 0.5 [13]. The vaccine coverage $f_{c}$ required to bring the basic reproduction number $R_{0}$ to below 1 with a vaccine efficacy $\alpha$ is given as [11]:

$$
f_{c}=\frac{1}{\alpha}\left(1-\frac{1}{R_{0}}\right)
$$

Given a value of $\alpha=0.5$ and the basic reproduction number $R_{0}=2.0$ during an epidemic, the theoretical vaccine coverage would be 1.0 , which will require $100 \%$ vaccination in the entire population and thus would exert immense financial and administrative strains on the economy. Fifth, the estimated cost of dengue illness per capita for Malaysia was merely USD 2.03 (2009) as given in Shepard et al. [12]. This cost would imply dengue cost of less than USD 4.00 (2016) per capita, adjusted for GDP of USD 6830 in 2009 and the recent GDP of about USD 11,000 for Malaysia. This benchmark fig. of USD 4.00 per capita should serve as a guide for highly cost-effective threshold for dengue vaccines in 2016. Using the fig. estimated above, this paper advocates that a highly cost-effective price threshold for dengue vaccine is about USD 5.00 for Malaysia. Given that the production cost of dengue vaccine is USD 0.50 per dose, this threshold price of USD 5.00 per dose would confer adequate profits to producers to recover their $\mathrm{R}$ and $\mathrm{D}$ costs plus a handsome profit.

\section{CONCLUSION}

In this paper, we propose a pharmacoeconomic model for dengue vaccine price valuation based upon the WHO guidelines that link the in-country threshold price to the GDP per capita. We opted to mandate that the price should be highly cost-efficient, as opposed to merely cost-effective, reflecting the MIC status of Malaysia. We take into consideration a realistic mosquito biting rate of 0.33 per female mosquito per day and other creditable input parameters that reflect the mosquito ecology. Taking into consideration a combination of five criteria, this paper concludes that a highly cost-effective price threshold in Malaysia for dengue vaccine is USD 5.00 per dose. This price threshold would be widely affordable to and readily adopted by the Malaysian consumers, which will ensure sustained and predictable consumption. This, in turn, would allow producers to fine-tune mass production in a large, predictable manner to reduce the overall costs of production and distribution to less than USD 1.00 per dose. This will be a win-win situation for both producers and consumers. Further research to develop a transparent and comprehensive cost-effective pricing model for dengue vaccines will be pursued in the near future. 
This pharmacoeconomic model will be based upon a comprehensive DENV dynamic transmission model that connects human SIR transmission framework [15] to mosquito ecology model [16] and that is linked to a pricing model as demonstrated in Shepard et al.[12], Shafie [13] and Coudeville and Garnett [14]

\section{ACKNOWLEDGEMENT}

Financial support provided by USM Research University grant \#1001/PMATHS/8011018 is gratefully acknowledged.

\section{CONFLICTS OF INTERESTS}

All authors have none to declare

\section{REFERENCES}

1. HL Koh. SY the FluSiM simulation for Malaysia: towards improved pandemic surveillance. Int J Chem Eng Appl 2011;2:53-9.

2. YA Halasa, DS Shepard, W Zeng. Economic cost of dengue in puerto rico. Am J Trop Med Hyg 2012;86:745-52.

3. WHO. Global strategy for dengue prevention and control 2012 2020. Geneva: World Health Organization; 2012.

4. JA Suaya, DS Shepard, JB Siqueira, CT Martelli, LCS Lum, LH Tan, et al. Cost of dengue cases in eight countries in the Americas and Asia: a prospective study. Am J Trop Med Hyg 2009; 80:846-55.

5. DS Shepard, EA Undurraga, YA Halasa. Economic and disease burden of dengue in Southeast Asia. PLoS Negl Trop Dis 2013;7:1-12.

6. RT Mahoney, DP Francis, NM Frazatti-Gallin, AR Precioso, I Raw, Watler $\mathrm{P}$, et al. Cost of production of live attenuated dengue vaccines: a case study of the Instituto Butantan, Sao Paulo, Brazil. Vaccine 2012;30:4892-6.
7. J Halstead, SB Deen. The future of dengue vaccines. Lancet 2002;360:1234-45.

8. M Kaddar, S Schmitt, M Makinen, J Milstien. Global support for new vaccine implementation in middle-income countries. Vaccine 2013;31 Suppl 2:B81-B96.

9. S Ozawa, A Mirelman, ML Stack, DG Walker, OS Levine. Costeffectiveness and economic benefits of vaccines in low and middle-income countries: a systematic review. Vaccine 2012;31:96-108.

10. ME Beatty, P Beutels, MI Meltzer, DS Shepard, J Hombach, R Hutubessy, et al. Health economics of dengue: a systematic literature review and expert panel's assessment. Am J Trop Med Hyg 2011;84:473-88.

11. LR Carrasco, LK Lee, VJ Lee, EE Ooi, DS Shepard, TL Thein, et al Economic impact of dengue illness and the cost-effectiveness of future vaccination programs in Singapore. PLoS Neglected Trop Dis 2011;5:e1426.

12. DS Shepard, EA Undurraga, RS Lees, Y Halasa, LCS Lum, CW Ng Use of multiple data sources to estimate the economic cost of dengue illness in Malaysia. Am J Trop Med Hyg 2012;87:796-805.

13. Shafie. Cost effective price of dengue vaccination in Malaysia. Personal communication; 2016.

14. L Coudeville, GP Garnett. Transmission dynamics of the four dengue serotypes in southern Vietnam and the potential impact of vaccination. PLoS One 2012;7:e51244.

15. HL Koh, SY Teh, DL De Angelis, J Jiang. Infectious diseases: surveillance, genetic modification and simulation. In: CA Brebbia, AJ Kassab, EA Divo. Editors. Disaster Management and Human Health Risk II. Boston: WIT Press; 2011. p. 245-256.

16. SC Chen, MH Hsieh. Modelling the transmission dynamics of dengue fever: Implications of temperature effects. Sci Total Environ 2012;431:385-91 